\title{
Relation of red cell distribution width with HAS-BLED score in patients with non-valvular atrial fibrillation
}

\author{
Hong-Li Cai", Hao Chen", Jing Wang, Ling Xie, Kou-Long Zheng, Qing Zhang \\ The Affiliated Hospital 2 of Nantong University, Nantong, China \\ Contributions: (I) Conception and design: Q Zhang, HL Cai; (II) Administrative support: KL Zheng; (III) Provision of study materials or patients: J \\ Wang; (IV) Collection and assembly of data: L Xie; (V) Data analysis and interpretation: H Chen; (VI) Manuscript writing: All authors; (VII) Final \\ approval of manuscript: All authors. \\ "These authors contributed equally to this work. \\ Correspondence to: Qing Zhang, PhD. Department of General practice, Affiliated Hospital 2 of Nantong University, Nantong 226001, China. \\ Email: zzhangqing32@sina.cn; Kou-Long Zheng, PhD. Department of Cardiology, Affiliated Hospital 2 of Nantong University, Nantong 226001, \\ China. Email: zklsir@126.com.
}

\begin{abstract}
Background: Numerous researchers have shown that there is a close correlation between red cell distribution width (RDW) and cardiovascular disease such as heart failure, coronary heart disease, and atrial fibrillation. This study was designated to investigate the correlation between RDW and the Hypertension, Abnormal renal/liver function, Stroke, Bleeding history or predisposition, Labile international normalized ratio, Elderly, Drugs/alcohol (HAS-BLED) score.

Methods: The HAS-BLED scores of 251 hospitalized patients with non-valvular atrial fibrillation were calculated and the receiver operating characteristics were used to evaluate the predictive value of RDW on high HAS-BLED score ( $\geq 3$ scores). Multiple logistic regression analysis was used to analyze the independent predictor of high HAS-BLED scores.

Results: Correlation analysis between RDW and HAS-BLED scores showed the RDW was positively correlated with HAS-BLED score, with $\mathrm{r}=0.393(\mathrm{P}<0.0001)$. The RDW of the high HAS-BLED score group was higher than that of the no-high HAS-BLED score group. The area under the receiver operating characteristic curve of RDW was $0.796(0.740-0.844, \mathrm{P}<0.0001)$ to predict a high HAS-BLED score, and multiple logistic regression analysis showed that a high RDW value could be used as an independent predictor of high HAS-BLED.
\end{abstract}

Conclusions: RDW value is associated with HAS-BLED value, and can be used as the independent predictive factor of high HAS-BLED scores.

Keywords: Red cell distribution width (RDW); atrial fibrillation; HAS-BLED score

Submitted Mar 01, 2021. Accepted for publication Apr 29, 2021.

doi: $10.21037 /$ jtd-21-567

View this article at: http://dx.doi.org/10.21037/jtd-21-567

\section{Introduction}

Atrial fibrillation is an independent risk factor of cerebral ischemic stroke and anticoagulant therapy is particularly important for the prevention of cerebral arterial thrombosis $(1,2)$. Strategies for antithrombotic therapy are made according to the thromboembolism and bleeding risk evaluation of atrial fibrillation patients, with the CHA2DS2-VASc score the most common scoring system evaluating thromboembolism risk in patients with atrial fibrillation (3). In 2010, the anticoagulation bleeding risk scoring system (HAS-BLED) was firstly proposed in ESC guidelines for the management of atrial fibrillation (4). The red cell distribution width (RDW) mainly reflects the nonuniformity of red blood cell volume in peripheral blood and in the past, has mainly been used in the clinical evaluation of hematological system diseases (5). Research 
Table 1 HAS-BLED score

\begin{tabular}{lc}
\hline Risk factors & Score \\
\hline H (hypertension) & 1 \\
A (abnormal renal/liver function) & 1 or 2 \\
S (stroke) & 1 \\
B (bleeding history or predisposition) & 1 \\
L (labile international normalized ratio) & 1 \\
E (elderly, N>65) & 1 \\
D (drugs/alcohol concomitantly) & 1 or 2 \\
\hline
\end{tabular}

has shown that RDW was associated with the prognosis of cardiovascular disease such as heart failure, coronary heart, disease and atrial fibrillation (6-9) and with some components of major scoring systems such as those used in stroke and hypertension $(10,11)$. In the absence of clinical information to calculate embolism or bleeding scores, a convenient index with a high predictive value for CHA2DS2-VASc or HAS-BLED scores, stroke, and bleeding risk would hold high clinical utility. While Kurt et al. (12) showed that the RDW value could be used as an independent predictive factor for high CHA2DS2-VASc scores $(\geq 2)$, to date, there has been no research reporting the relationship between RDW and HAS-BLED, which is the objective of this study.

We present the following article in accordance with the STARD reporting checklist (available at http://dx.doi. org/10.21037/jtd-21-567).

\section{Methods}

\section{Study population:}

We selected 251 non-valvular atrial fibrillation patients hospitalized between January 2014 and January 2015 in the cardiovascular department of the Second Affiliated Hospital of Nantong University, of which there were 129 males and 122 females with an average age of $67.15 \pm 7.55$ years. Other factors recorded at admission included blood pressure, history of past or concomitant disease such as hypertension, diabetes, cerebral ischemic or stroke, bleeding history, past drinking history, international normalized ratio (INR) labile history, and non-steroid anti-inflammatory and antiplatelet drug use. Blood samples were collected on an empty stomach in the morning the day after hospitalization to test the following: RDW, hematocrit value, mean corpuscular hemoglobin concentration, mean corpuscular hemoglobin, mean corpuscular volume, hemoglobin, platelet count, mean platelet volume, platelet distribution width, and alanine aminotransferase, total bilirubin, serum creatinine, cystatin, and N-terminal pro brain natriuretic peptide (NTproBNP) levels. The exclusion criteria for this study were the presence or history of valvular atrial fibrillation, serious hepatic and renal dysfunction, blood disease, malignancy, active bleeding, thyroid dysfunction, chronic rheumatic immune disease, recent infection, surgical operation, and blood transfusion.

Atrial fibrillation was defined as absolutely irregular RR intervals and no discernible, distinct $\mathrm{P}$ waves (1). We evaluated the risk of bleeding with HAS-BLED score using specific scoring rules which included (4) hypertension, abnormal renal/liver function, stroke, bleeding history or predisposition, labile international normalized ratio, elderly ( $>$ $>65$ ), and drugs/alcohol concomitantly (Table 1), with a score of " $\geq 3$ " recognized as a high bleeding risk. We also employed a HAS-BLED score that is used to estimate the risk of long-term bleeding events associated with atrial fibrillation in which patients scoring " $\geq 3$ " were allocated into a high-risk group and those with a score of " $<3$ " were allocated to a no-high risk group.

The mean corpuscular hemoglobin, mean corpuscular hemoglobin concentration, mean corpuscular volume, RDW, hemoglobin, platelet count, mean platelet volume, and platelet distribution width were determined by the automated hematology analyzer XE-2100 (Sysmex, Kobe, Japan), while the serum creatinine, alanine aminotransferase, cystatin, and total bilirubin levels were determined using a Hitachi Automatic Analyzer 7600 (Hitachi, Tokyo, Japan). The NT-proBNP level was measured by electrochemiluminescence immunoassay on the Dimension Vista 500 Intelligent Laboratory System (Siemens Healthcare Diagnostics, Deerfield, Illinois, United States). Finally, the normal reference range for RDW was set at $11.5 \%$ to $14.5 \%$.

All procedures performed in this study involving human participants were in accordance with the Declaration of Helsinki (as revised in 2013). The study was approved by the Institutional ethics Committee of the Second Affiliated Hospital of Nantong University (No. 2020KN088) and informed consent was taken from all the patients.

\section{Statistical analysis}

The measurement data were presented as means \pm standard 
Table 2 Comparison of the general clinical information between the two groups according to HAS-BLED score

\begin{tabular}{|c|c|c|c|}
\hline Variables & No-high risk $(n=205)$ & High risk $(n=46)$ & $P$ value \\
\hline Female, n (\%) & $95(46.3)$ & $27(58.6)$ & 0.130 \\
\hline Hypertension, n (\%) & $85(41.5)$ & $29(63.0)$ & 0.008 \\
\hline Type II diabetes, n (\%) & $31(15.1)$ & $8(17.4)$ & 0.701 \\
\hline Bleeding history, n (\%) & $8(3.9)$ & $1(2.2)$ & 0.569 \\
\hline Labile INR, n (\%) & $10(4.9)$ & $3(6.5)$ & 0.649 \\
\hline Drugs/alcohol use concomitantly, n (\%) & $18(8.7)$ & $8(17.4)$ & 0.083 \\
\hline Hematocrit & $0.411 \pm 0.437$ & $0.371 \pm 0.438$ & 0.000 \\
\hline MCV (fL) & $90.61 \pm 5.03$ & $90.60 \pm 4.95$ & 0.993 \\
\hline RDW (\%) & $13.08 \pm 1.03$ & $13.96 \pm 0.93$ & 0.000 \\
\hline Hemoglobin (g/L) & $137.38 \pm 15.37$ & $121.80 \pm 14.31$ & 0.000 \\
\hline PLT (×109\%/L) & $189.31 \pm 53.03$ & $174.37 \pm 51.28$ & 0.154 \\
\hline MPV (fL) & $10.88 \pm 1.21$ & $11.13 \pm 1.41$ & 0.336 \\
\hline PDW (\%) & $13.58 \pm 2.83$ & $14.00 \pm 3.49$ & 0.478 \\
\hline NT-ProBNP (ng/L) & $364.51 \pm 659.72$ & $673.89 \pm 811.08$ & 0.084 \\
\hline ALT (U/L) & $23.31 \pm 25.39$ & $27.65 \pm 20.06$ & 0.403 \\
\hline
\end{tabular}

Drugs use concomitantly: concomitant use of non-steroidal anti-inflammatory drugs (NSAIDs)/antiplatelet drugs. INR, international normalized ratio; $\mathrm{MCH}$, mean corpuscular hemoglobin; $\mathrm{MCHC}$, mean corpuscular hemoglobin concentration; MCV, mean corpuscular volume; RDW, red cell distribution width; PLT, platelet count; MPV, mean platelet volume; PDW, platelet distribution width; NT-ProBNP, N terminal pro B type natriuretic peptide; ALT, alanine aminotransferase; Tb, total bilirubin; Scr, serum creatinine.

deviation, while the enumeration data were presented as percentage or frequency. Independent-sample $t$-test and chisquare test were applied in comparing the measurement data and the enumeration data in the two groups, respectively, and bivariate Spearmen was used for the correlation analysis. The predictive values of RDW on a high HAS-BLED score was completed through receiver-operating characteristic (ROC) analyses and multiple logistic regression analysis was used to evaluate the relationship between variables and a high HAS-BLED score. Variables with a $\mathrm{P}$ value $<0.1$ in univariate logistic regression analysis were included in a multivariate logistic regression model and the results of the regression analysis were presented as odds ratios and $95 \%$ confidence intervals. The data analysis was completed with SPSS 17.0 (SPSS Inc., Chicago, Illinois, USA) and MedCalc (version 11.2.1; MedCalc, Mariakerke, Belgium) and a difference of $\mathrm{P}<0.05$ was deemed as statistically significant.

\section{Results}

Comparison between the characteristics in the two groups showed hypertension (\%), stroke (\%), RDW, and cystatin were higher in the high-risk group than the no-high risk group $(\mathrm{P}<0.05)$, while the hematocrit and hemoglobin levels were lower than those in the no-high risk group $(\mathrm{P}<0.05)$ (Table 2). 


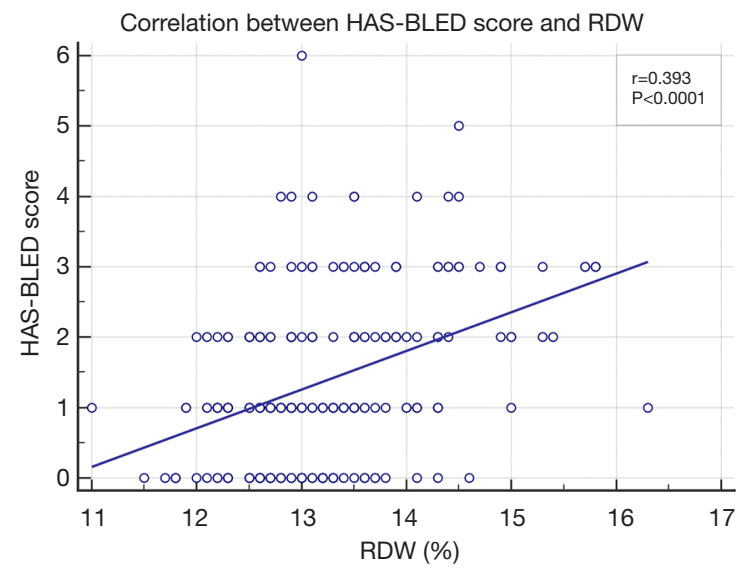

Figure 1 Correlation analysis between RDW and HAS-BLED scores showed the RDW was positively correlated with HASBLED score, with $\mathrm{r}=0.393(\mathrm{P}<0.0001)$. RDW, red cell distribution width.

Correlation analysis between RDW and HAS-BLED scores showed the RDW was positively correlated with HAS-BLED score, with $\mathrm{r}=0.393(\mathrm{P}<0.0001)$ (Figure 1), while the area under the ROC curve of RDW to predict a high HAS-BLED score was 0.796 , with the cut-off level of $13.3 \%, 95 \%$ CI of $0.740-0.844$, sensitivity of $72.5 \%$, and specificity of $73.3 \%(\mathrm{P}<0.0001)$ (Figure 2$)$.

Multiple logistic regression analysis showed that RDW (OR: 1.33, 95\% CI: 1.10-1.56, $\mathrm{P}=0.031$ ), stroke (OR: 3.13, 95\% CI: 1.65-4.35, $\mathrm{P}=0.003$ ), hemoglobin (OR: 0.93, 95\% CI: $0.88-0.98, \mathrm{P}=0.040$ ), and hypertension (OR: $2.30,95 \%$ CI: $1.25-3.99, \mathrm{P}=0.022)$ were independent predictors of a high HAS-BLED score (Table 3).

\section{Discussion}

The results showed that the RDW value in the high HASBLED score group was significantly higher than in the nohigh HAS-BLED score group and was positively correlated with a HAS-BLED score. In addition, RDW could be used as an independent predictive factor of high HAS-BLED scores.

Atrial mural thrombi can cause cerebral embolism and stroke, and patients with atrial fibrillation have a higher fatality rate, invalidism rate, and hospitalization stay duration than those without. Different strategies for anticoagulation management may be used according to the evaluation of CHA2DS2-VASc scores on thromboembolism risk. RDW reflects the dispersion degree of red blood cells

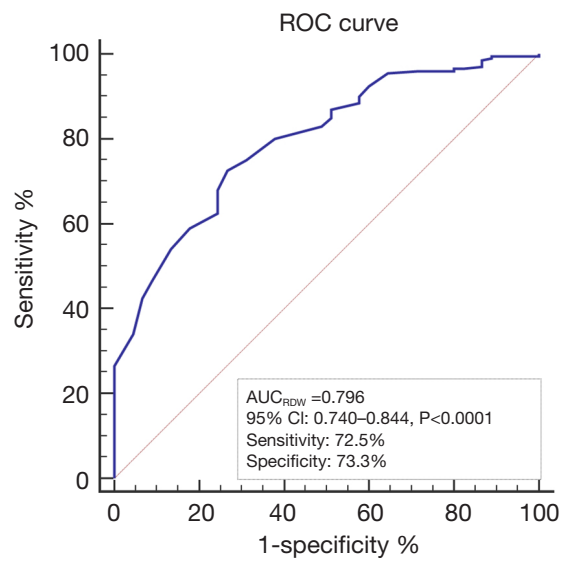

Figure 2 The ROC AUC of RDW in predicting a high HASBLED score was 0.796 , with the cut-off level of $13.3 \%$, 95\% CI of $0.740-0.844$, sensitivity of $72.5 \%$, and specificity of $73.3 \%$ $(\mathrm{P}<0.0001)$. RDW, red cell distribution width. ROC, receiveroperating characteristic; AUC, area under the curve.

Table 3 Multiple logistic regression analysis to detect the independent predictors of high HAS-BLED score in patients with AF

\begin{tabular}{lcc}
\hline Variables & OR, 95\% Cl & P value \\
\hline Hemoglobin & $0.93(0.88-0.98)$ & 0.040 \\
RDW & $1.33(1.10-1.56)$ & 0.031 \\
Hypertension & $2.30(1.25-3.99)$ & 0.022 \\
Stork & $3.13(1.65-4.35)$ & 0.003 \\
\hline
\end{tabular}

RDW, red cell distribution width; $A F$, atrial fibrillation.

in peripheral blood and in the past, was mainly used for the morphological classification of anemia including the differential diagnosis of iron-deficiency anemia clinically (13). Lee et al. (9) showed that the incidence rate of CHA2DS2VASC scores and cerebral arterial thrombosis increased with an increasing RDW value. While preventing thrombus, bleeding risk should also be evaluated in the process of using anticoagulant drugs and at present, the HAS-BLED scoring system is the most widely used scoring system. Research has shown that RDW was also correlated to HAS-BLED scores, and can be used as an independent predictive factor of high HAS-BLED scores.

An increased RDW has been shown to be associated with high blood pressure and could be used as a predictive factor of high blood pressure organ damage (14). A largescale cohort study of Hoffmann (15) showed that RDW was age dependent and tended to increase with age, while Lippi 
et al. (16) studied RDW and creatinine values in a large sample of outpatients and found that RDW was negatively correlated with renal function. In addition, correlation between high RDW values with abnormal liver function and ischemic stroke has been demonstrated in several studies (17-19). High blood pressure, age, abnormal hepatorenal function, and stroke are the major factors which constitute the HAS-BLED score system and this may explain the predictive value of RDW on high HAS-BLED scores. At present, an increase in the RDW value is believed to be correlated with factors such as a lack of nutrients, including folic acid and vitamin B12, which cause anemia, and the resulting changes to hemopoietin cause impaired renal function, activation of the neuroendocrine system, oxidative stress, and in vivo neuroendocrine system changes (20-26).

\section{Limitations}

The major limitations to this study are its small sample size, short follow-up period, and that it was limited only to the correlation between RDW and HAS-BLED.

\section{Conclusions}

RDW values are easy to obtain, have a strong predictive value for high HAS-BLED scores, and can be used as an auxiliary index to evaluate bleeding risk in the process of atrial fibrillation anticoagulation therapy.

\section{Acknowledgments}

We acknowledge the assistance of all doctors, nurses, technicians, ambulance paramedics, and hospital staff who cared for our patients.

Funding: None.

\section{Footnote}

Reporting Checklist: The authors have completed the STARD reporting checklist. Available at http://dx.doi.org/10.21037/ jtd-21-567

Data Sharing Statement: Available at http://dx.doi. org/10.21037/jtd-21-567

Conflicts of Interest: All authors have completed the ICMJE uniform disclosure form (available at http://dx.doi. org/10.21037/jtd-21-567). The authors have no conflicts of interest to declare.

Ethical Statement: The authors are accountable for all aspects of the work in ensuring that questions related to the accuracy or integrity of any part of the work are appropriately investigated and resolved. All procedures performed in this study involving human participants were in accordance with the Declaration of Helsinki (as revised in 2013). The study was approved by the Institutional ethics Committee of the Second Affiliated Hospital of Nantong University (No. 2020KN088) and informed consent was taken from all the patients.

Open Access Statement: This is an Open Access article distributed in accordance with the Creative Commons Attribution-NonCommercial-NoDerivs 4.0 International License (CC BY-NC-ND 4.0), which permits the noncommercial replication and distribution of the article with the strict proviso that no changes or edits are made and the original work is properly cited (including links to both the formal publication through the relevant DOI and the license). See: https://creativecommons.org/licenses/by-nc-nd/4.0/.

\section{References}

1. Kirchhof P, Benussi S, Kotecha D, et al. 2020 ESC Guidelines for the Management of Atrial Fibrillation Developed in Collaboration with EACTS. Eur Heart J 2020;37:2893-962.

2. Wolf PA, Abbott RD, Kannel WB. Atrial Fibrillation as an Independent Risk Factor for Stroke: The Framingham Study. Stroke 1991;22:983-8.

3. Lip GY, Nieuwlaat R, Pisters R, et al. Refining Clinical Risk Stratification for Predicting Stroke and Thromboembolism in Atrial Fibrillation Using a Novel Risk Factor-Based Approach: The Euro Heart Survey on Atrial Fibrillation. Chest 2010;137:263-72.

4. Pisters R, Lane DA, Nieuwlaat R, et al. A Novel UserFriendly Score (Has-Bled) to Assess 1-Year Risk of Major Bleeding in Patients with Atrial Fibrillation: The Euro Heart Survey. Chest 2010;138:1093-100.

5. Demir A, Yarali N, Fisgin T, et al. Most Reliable Indices in Differentiation between Thalassemia Trait and Iron Deficiency Anemia. Pediatr Int 2002;44:612-6.

6. Tonelli M, Sacks F, Arnold M, et al. Relation between Red Blood Cell Distribution Width and Cardiovascular Event Rate in People with Coronary Disease. Circulation 2008;117:163-8. 
7. Cheng YL, Cheng HM, Huang WM, et al. Red Cell Distribution Width and the Risk of Mortality in Patients with Acute Heart Failure with or without Cardiorenal Anemia Syndrome. Am J Cardiol 2016;117:399-403.

8. Ferreira JP, Girerd N, Arrigo M, et al. Enlarging Red Blood Cell Distribution Width During Hospitalization Identifies a Very High-Risk Subset of Acutely Decompensated Heart Failure Patients and Adds Valuable Prognostic Information on Top of Hemoconcentration. Medicine (Baltimore) 2016;95:e3307.

9. Lee KH, Park HW, Cho JG, et al. Red Cell Distribution Width as a Novel Predictor for Clinical Outcomes in Patients with Paroxysmal Atrial Fibrillation. Europace 2015;17 Suppl 2:ii83-8.

10. Buyukkaya E, Erayman A, Karakas E, et al. Relation of Red Cell Distribution Width with Dipper and NonDipper Hypertension. Med Glas (Zenica) 2016;13:75-81.

11. Lappegård J, Ellingsen TS, Skjelbakken T, et al. Red Cell Distribution Width Is Associated with Future Risk of Incident Stroke. The Tromso Study. Thromb Haemost 2016;115:126-34.

12. Kurt M, Tanboga IH, Buyukkaya E, et al. Relation of Red Cell Distribution Width with Cha2ds2-Vasc Score in Patients with Nonvalvular Atrial Fibrillation. Clin Appl Thromb Hemost 2014;20:687-92.

13. Nalbantoğlu B, Guzel S, Buyukyalcin V, et al. Indices Used in Differentiation of Thalassemia Trait from Iron Deficiency Anemia in Pediatric Population: Are They Reliable? Pediatr Hematol Oncol 2012;29:472-8.

14. Chen L, Li Z, Li Y, et al. Red Cell Distribution Width and Inappropriateness of Left Ventricular Mass in Patients with Untreated Essential Hypertension. PLoS One 2015;10:e120300.

15. Hoffmann JJ, Nabbe KC, van den Broek NM. Effect of Age and Gender on Reference Intervals of Red Blood Cell Distribution Width (Rdw) and Mean Red Cell Volume (Mcv). Clin Chem Lab Med 2015;53:2015-9.

16. Lippi G, Targher G, Montagnana M, et al. Relationship between Red Blood Cell Distribution Width and Kidney Function Tests in a Large Cohort of Unselected

Cite this article as: Cai HL, Chen H, Wang J, Xie L, Zheng KL, Zhang Q. Relation of red cell distribution width with HASBLED score in patients with non-valvular atrial fibrillation. J Thorac Dis 2021;13(5):3070-3075. doi: 10.21037/jtd-21-567
Outpatients. Scand J Clin Lab Invest 2008;68:745-8.

17. Jia H, Li H, Zhang Y, et al. Association between Red Blood Cell Distribution Width (Rdw) and Carotid Artery Atherosclerosis (Cas) in Patients with Primary Ischemic Stroke. Arch Gerontol Geriatr 2015;61:72-5.

18. Kayadibi H, Sertoglu E, Uyanik M. Is High Rdw Really Closely Associated with Nafld? Eur J Gastroenterol Hepatol 2014;26:815.

19. Söderholm M, Borne Y, Hedblad B, et al. Red Cell Distribution Width in Relation to Incidence of Stroke and Carotid Atherosclerosis: A Population-Based Cohort Study. PLoS One 2015;10:e0124957.

20. Nagajothi N, Braverman A. Elevated Red Cell Distribution Width in the Diagnosis of Thrombotic Thrombocytopenic Purpura in Patients Presenting with Anemia and Thrombocytopenia. South Med J 2007;100:257-9.

21. Semba RD, Patel KV, Ferrucci L, et al. Serum Antioxidants and Inflammation Predict Red Cell Distribution Width in Older Women: The Women's Health and Aging Study I. Clin Nutr 2010;29:600-4.

22. Pascual-Figal DA, Bonaque JC, Manzano-Fernandez S, et al. Red Blood Cell Distribution Width Predicts NewOnset Anemia in Heart Failure Patients. Int J Cardiol 2012;160:196-200.

23. Weiss G, Goodnough LT. Anemia of Chronic Disease. N Engl J Med 2005;352:1011-23.

24. Park TS, Zambidis ET. A Role for the Renin-Angiotensin System in Hematopoiesis. Haematologica 2009;94:745-7.

25. Kato H, Ishida J, Imagawa S, et al. Enhanced Erythropoiesis Mediated by Activation of the ReninAngiotensin System Via Angiotensin Ii Type 1a Receptor. FASEB J 2005;19:2023-5.

26. Förhécz Z, Gombos T, Borgulya G, et al. Red Cell Distribution Width in Heart Failure: Prediction of Clinical Events and Relationship with Markers of Ineffective Erythropoiesis, Inflammation, Renal Function, and Nutritional State. Am Heart J 2009;158:659-66.

(English Language Editor: B. Draper) 